\title{
Produção do conhecimento científico de Enfermagem em Nefrologia
}

\author{
Scientific knowledge production in Nephrology Nursing \\ Producción del conocimiento científico de Enfermería en Nefrología
}

\section{Viviane Peixoto dos Santos Pennafort', Angelina Monteiro Furtado', Ana Virgínia de Melo Fialho', Thereza Maria Magalhães Moreira', Maria Célia de Freitas', Maria Veraci Oliveira Queiroz' \\ 'Universidade Federal do Ceará. Mestrado em Cuidados Clínicos em Saúde. Fortaleza, CE}

Submissão: 07/07/2009

Aprovação: 20/06/2010

\section{RESUMO}

Trata-se de uma pesQuisa bibliográfica, com abordagem Quantitativa, Que teve como objetivo conhecer a produção científica das teses e dissertações disponíveis no banco de dados do Centro de Estudos e Pesquisa em Enfermagem (CEPEn) e sua aplicabilidade na área de enfermagem em nefrologia. Foram encontrados 48 resumos de teses e dissertações no período de 1979 a 2007 . Os resultados apontam Que estas produções se concentram nas regiões Sudeste e Sul. Com relação ao referencial teórico-metodológico, os mais freeüentes foram: método de assistência de enfermagem e abordagem compreensiva; os sujeitos investigados foram em sua maioria, os pacientes renais crônicos. Essa temática ainda é pouco investigada nos cursos de pós-graduação stricto senso, entretanto, há indícios de expansão dos estudos científicos nesta área.

Descritores: Conhecimento; Pesquisa em enfermagem; Diálise renal; Insuficiência renal crônica.

\section{ABSTRACT}

This is a bibliographic research, with Quantitative approach, that aimed to know the scientific production of theses and dissertations available in the database of the Center of Studies and Research in Nursing (CEPEn) and its applicability in the nursing area in nephrology. It was found 48 abstracts of theses and dissertations in the period from 1979 to 2007 . The results point that these productions are concentrated in the Southeast and Southern regions of Brazil. Regarding the theoretical-methodological referential, the most freeuent were: nursing assistance method and comprehensive approach; the investigated individuals were mostly the chronic renal patients. This thematic is still little investigated in the post-graduation stricto senso courses, however there are signs of expansion of the scientific studies in this area.

Key words: Knowledge; Nursing research; Renal dialysis; Renal insufficiency, chronic.

\section{RESUMEN}

Se trata de una búsqueda bibliográfica con abordaje cuantitativo, con el objetivo de conocer la producción científica de tesis y disertaciones disponibles en la base de datos del Centro de Estudios e Investigación en Enfermería (CEPEn) y su aplicabilidad en el campo de la enfermería en nefrología. Se han encontrado 48 resúmenes de tesis y disertaciones en el período 1979 a 2007. Los resultados indican Que estos producciones se concentran en el Sudeste y Sur del Brasil. En lo que respecta al marco teórico y a la metodología, los más frecuentes fueron: método de atención de asistencia de enfermería y abordaje comprensiva; los sujetos investigados fueron en su mayoría, los pacientes renales crónicos. Este tema ha sido poco investigado en el postgrado stricto sensu, no obstante, hay pruebas de la ampliación de los estudios científicos en este ámbito.

Descriptores: Conocimiento; Investigación en enfermería; Diálisis renal; Insuficiencia renal crónica.

\footnotetext{
AUTOR CORRESPONDENTE Viviane Peixoto dos Santos Pennafort. Rua Clóvis Meton, 189, Bairro Maraponga. CEP 607 I I-400. Fortaleza, CE. E-mail: vivipspf@yahoo.com.br
} 


\section{INTRODUÇÃO}

A pesQuisa em enfermagem vem ganhando espaço e importância no âmbito da produção e divulgação do conhecimento, a Qual é disponibilizada à comunidade científica, acadêmica e sociedade em geral. Inegavelmente, o aumento das pesQuisas surge com a ampliação dos cursos de pós-graduação Que tem o acompanhamento e orientação da Coordenação de Aperfeiçoamento de Pessoal de Nível Superior (CAPES). Nesse sentido, uma grande vitória na história da enfermagem foi o fato de ter sido reconhecida com Linha de Pesquisa no Fórum Nacional de Coordenadores de Cursos de Pós-graduação em Enfermagem, promovido em 2000 pela CAPES. Isto legitima enquanto área importante de investigação(l).

No Brasil, os anos 60 e 70 foram marcados pelo desenvolvimento teórico da profissão com o surgimento do primeiro programa de pós-graduação stricto sensu em Enfermagem no Brasil em 1972 na Escola de Enfermagem Ana Nery da Universidade Federal do Rio de Janeiro, sendo o primeiro doutorado autorizado somente em $1980^{(2)}$

Diante desta perspectiva(3) ${ }^{(3)}$ o significativo desenvolvimento da pós-graduação a partir da década de 80 , foi inegável a ampliação da produção científica na área de enfermagem, tanto do ponto de vista de dissertações e teses como de outras produções científicas. Atualmente a pós-graduação stricto sensu é um seguimento consolidado no cenário educacional brasileiro e internacional, na área da saúde e da enfermagem. Ressalta ainda ${ }^{(4)}$ a contribuição do repensar e reconstruir na Qualificação em enfermagem, à medida Que, subsidia uma atitude reflexiva em torno dos significados e das tecnologias no cuidado humano.

Considerando Que a assistência de enfermagem é uma atividade fundamental para a formação profissional do enfermeiro, o estudo da produção científica das teses e dissertações na área da enfermagem em nefrologia busca entender como estas pesquisas exercem influência na práxis da enfermagem junto ao paciente com Insuficiência Renal Crônica Terminal (IRCT).

Sabe-se Que a Insuficiência Renal Crônica (IRC) geralmente se desenvolve após uma injúria renal inicial Que é seguida de perda lenta, progressiva e irreversível da função renal. Em sua fase mais avançada, chamada de fase terminal, os rins não conseguem mais manter as suas funções regulatórias, excretórias e endócrinas. $\mathrm{O}$ diagnóstico da IRC baseia-se na identificação dos grupos de risco, presença de microalbuminúria, proteinúria, hematúria e na redução do ritmo de filtração glomerular (RFG) avaliado por um teste laboratorial chamado clearance da creatinina sérica ${ }^{(5)}$.

O tratamento da IRC inclui duas etapas: I - O tratamento conservador, antes da necessidade da terapia de substituição da função renal; 2 - Terapia de substituição renal (TSR), incluindo as diversas modalidades de diálise e o transplante renal. Considerando o censo 2008 da Sociedade Brasileira de Nefrologia ${ }^{(6)}$ : no mundo, cerca de 1 milhão e 200 mil pessoas sobrevivem sob tratamento hemodialítico. No Brasil são aproximadamente 87.044 pacientes por ano, dos Quais $89,4 \%$ estão em tratamento hemodialítico e 10,6\% em diálise peritoneal.

Diante deste contexto, devido ao aumento da demanda da Terapia Renal Substitutiva (TRS) a Sociedade Brasileira de Nefrologia (SBN) considera que a tragédia de Caruaru, ocorrida no Instituto de Doenças Renais na cidade de mesmo nome no estado de Pernambuco, marcou a história da diálise brasileira Quando em 1996, morreram 54 pacientes renais vitimados pela contaminação da água. Diante da importância epidemiológica da doença renal, bem como suas repercussões sociais, psicológicas, econômicas e de baixa Qualidade de vida, o Ministério da Saúde edita portarias com regulamentações e normas técnicas, visando uma melhoria na Qualidade do atendimento ao cliente Que necessita de diálise ${ }^{(5)}$.

Analisando o processo histórico da Enfermagem, nota-se uma vertiginosa influencia, da profissão, pelos ideais positivistas de Comte (1789-1857) desde o século XIX até a gênese da ciência no Brasil no século XX. É oportuno salientar Que essa enfermagem moderna do século XX e início do século XXI segue os padrões propostos por Florence Nightingale: a noção do "dever de todos para com todos"; a fórmula "viver para outrem", onde se verifica uma preponderância dos instintos altruístas sobre os egoístas; e ainda, a concepção biologicista do homem/ambiente/doença ${ }^{(7)}$.

Acompanhando essa construção histórica, é no século XXI Que a enfermagem brasileira se insere no universo das terapias renais substitutivas e no transplante renal, se integrando a uma clínica permeada pela expansão dos equipamentos tecnológicos, da tecnologia dura, distanciando as relações subjetivas do cuidado ao paciente renal crônico. Estudos ${ }^{(8)}$ mostram Que desde a década de 60 , o enfermeiro inserido no serviço de diálise tem ampliado o seu campo de atuação, exercendo diversificadas funções, a saber: a) assistencial, proporcionando ao paciente um tratamento dialítico eficiente; b) social, ensino, pesquisa, gerenciamento, responsabilidade legal; c) interdependência, mantendo, promovendo e recuperando a saúde, junto aos demais integrantes da equipe multidisciplinar.

Diante do exposto, este trabalho teve como objetivo: identificar a produção científica das teses e dissertações disponíveis nos catálogos do Centro de Estudos e Pesquisa em Enfermagem (CEPEn) e sua aplicabilidade na área de enfermagem em nefrologia.

\section{METODOLOGIA}

Tratou-se de uma pesquisa bibliográfica. O material para a consolidação da pesquisa bibliográfica já deve ter sido publicado e se constitui principalmente de livros, artigos de periódicos e atualmente de material disponibilizado na Internet ${ }^{(9)}$.

A fonte de dados da pesquisa foram os resumos publicados nos catálogos do Centro de Estudos e Pesquisa em Enfermagem (CEPEn) da Associação Brasileira de Enfermagem (ABEn). Os catálogos estão disponíveis em CD-ROM e divididos em dois períodos: CD 75 Anos da ABEn (1979 a 2000) e CD 80 Anos da ABEn (200I a 2007) - também disponível no site da ABEn. A busca aconteceu nos meses de outubro e novembro, através da consulta dirigida pelo índice de assunto dos catálogos do CEPEn, foram utilizadas as seguintes palavras-chave: insuficiência renal crônica, diálise, diálise renal e hemodiálise.

O CEPEn, foi criado em 17 de julho de 1971, é destinado a incentivar o desenvolvimento e a divulgação da pesquisa em enfermagem, organizar e preservar documentos históricos da profissão e rege-se pelas disposições do Estatuto da ABEn e de Estatuto Especial. Possui o maior banco de teses e dissertações 
na área de Enfermagem no Brasil, hoje com mais de 4000 trabalhos registrados em seu acervo, além de possuir Quase todos os títulos de periódicos brasileiros de enfermagem ${ }^{(10)}$. Nesse contexto, o CEPEn ao disponibilizar seu acervo científico, está contribuindo para a socialização do conhecimento produzido pela enfermagem, e ainda, para a construção da história da enfermagem brasileira.

Foram encontrados 48 resumos referentes às teses $\mathrm{e}$ dissertações disponíveis no CEPEn, no período de 1979 a 2007. Como critério de inclusão considerou-se os resumos pertinentes ao objetivo do estudo, dentro deste corte temporal; como critério de exclusão não foi contemplado para análise os resumos referentes à temática Que abordava a Insuficiência Renal Aguda, pois o contexto da assistência de enfermagem nesta condição é significativamente distinto do processo crônico Que envolve a Insuficiência Renal Crônica, já Que este acontece de forma permanente.

Diante desses critérios, a amostra do estudo correspondeu a 46 resumos Que foram submetidos às seguintes etapas: leitura e preenchimento do formulário, construção de banco de dados e análise.

A leitura do material foi orientada por um roteiro Que contemplava os aspectos relacionados aos objetivos da pesquisa. Foram considerados como critérios de caracterização do estudo: a abordagem metodológica do estudo, o seu referencial teóricometodológico, a região onde foi desenvolvida, a fonte de coleta de dados e/ou participantes do estudo, a área temática e a sua aplicabilidade na enfermagem, a modalidade terapêutica em nefrologia e o ano de defesa das teses e dissertações.

As informações foram armazenadas em banco de dados informatizado com apoio do programa Microsoft Excel. A construção deste banco de dados correspondeu à etapa de organização dos fenômenos coletados, visando contemplar as informações relevantes e inerentes ao objeto de estudo desta pesquisa. Inicialmente, fizemos o rol, ou seja, a ordenação dos dados brutos, partindo, então, para a construção das séries estatísticas. O agrupamento dos dados contidos nas séries estatísticas encontradas considerou a Quantidade de elementos, o número de repetições e o tipo de variável.

A análise dos dados foi frequencial e a sua apresentação em forma de gráficos e Quadros, sendo, os mesmos discutidos de acordo com material bibliográfico concernente às tendências na produção do conhecimento científico na enfermagem, bem como de reflexões acerca da aplicabilidade e contribuição desse conhecimento para a enfermagem atuante na área da nefrologia.

\section{RESULTADOS E DISCUSSÃO}

A evolução histórica da Enfermagem evidencia a disposição de seus exercentes, numa busca permanente para a prestação de um cuidado de Qualidade ao paciente. No inicio, esse cuidado era ministrado com característica empírica, baseado em normas e rotinas repetidas, sem reflexão crítica e registro das ações executadas. Na necessidade de Questionar esse seu modo de agir tecnicamente e intuitivamente orientado, a Enfermagem teve Que refletir sobre a eficiência de seus métodos e práticas cotidianas, atribuindo maior importância à aplicação de princípios científicos Que pudessem conferir cientificidade à profissão.
Nesse sentido, o conhecimento científico de enfermagem enunciado da análise dos resumos das teses e dissertações Que rege este trabalho propõe-se agora a ser reconstruído. Para tanto, devemos lembrar Que conhecemos com base no Que já está conhecido. Sendo a construção do conhecimento preterida a sua reconstrução porQue devemos retomar o conhecimento disponível e o refazermos com a autenticidade inerente ao ato de pesquisar e elaborar ${ }^{(11)}$.

A primeira variável a ser apresentada é a abordagem metodológica das pesquisas, verificou-se Que houve uma aproximação estatística entre as abordagens Quantitativas (52\%) e Qualitativas (46\%), demonstrando a importância e aplicabilidade das diferentes naturezas de pesquisa. Apareceu também a pesquisa ClínicoQualitativa ${ }^{(12)}$ Que é sustentada por três pilares: a atitude existencialista da valorização dos elementos; a atitude clínica da acolhida dos sofrimentos emocionais das pessoas. Inclinando-lhe a escuta e o olhar; e atitude psicanalítica da dinâmica do inconsciente do indivíduo.

Quanto ao referencial teórico presente no Quadro I das teses e dissertações, infere-se Que o conhecimento da enfermagem flexibilizou a aproximação com as ciências humanas. Consequentemente, possibilitou a aquisição de habilidades na utilização de outras referências teóricas metodológicas, tais como, a abordagem compreensiva, representções sociais, sociopoética e etnometodologia.

Levando-se em consideração o método de assistência de enfermagem, como terceiro referencial teórico mais utilizado, notase uma preocupação de mudança na organização do processo de trabalho desse enfermeiro. O método de assistência de enfermagem engloba o processo de enfermagem e o uso de teorias na sistematização dessa assistência ${ }^{(13)}$. Contudo, a utilização de diferentes trajetórias metodológicas e marcos conceituais demonstram a amplitude de abordagens e fundamentações possíveis à prática da enfermagem em nefrologia.

Com relação ao ano de defesa, houve um aumento na produção científica das teses e dissertações no decorrer dos períodos analisados, sendo Que até 1988 a produção científica na área de enfermagem em nefrologia correspondia a $4 \%$ do total de resumos analisados. Somente no final da década de 90 Que esta produção tornou-se mais expressiva.

Ao analisarmos os indicadores sobre o ensino de pós-graduação do Ministério da Ciência e Tecnologia ${ }^{(14)}$, verificamos uma evolução vertiginosa e perene nos cursos de mestrado e doutorado brasileiros na última década. Fato este congruente com os dados coletados, todavia é relevante ressaltar Que neste período a enfermagem em nefrologia passou por transformações na sua atuação e formação, decorrentes da Tragédia de Caruaru em 1996, fato este de repercussão internacional, diante disto, o Ministério da Saúde estabeleceu o Regulamento Técnico para o funcionamento dos serviços de diálise e as normas para cadastramento destes junto ao Sistema Único de Saúde através da portaria 082 de 03 de janeiro de 2000.

Entre 2004 e 2007 esta produção correspondeu a 39\% do total, o Que vale dizer Que existe uma tendência de expansão de estudos em enfermagem nefrológica nos últimos anos. Esse fato vem se intensificando na enfermagem com a regulamentação da pós-graduação stricto-sensu, e mais recentemente ainda Quando 


\begin{tabular}{|l|c|}
\hline Referencial teórico-metodológico & $\mathbf{n}$ \\
\hline Método de Assistência de Enfermagem & 8 \\
Abordagem Compreensiva & 13 \\
Etnometodologia & 2 \\
Integralidade e Promoção da Saúde & 2 \\
Lócus de Controle de Rotter e Aderência & 2 \\
PesQuisa-ação & 1 \\
PesQuisa Convergente Assistencial & 1 \\
Teoria do Cuidado de Mayeraff & 1 \\
Teorias das crises de Caplan e Levy & 1 \\
Representação Social & 1 \\
Sociopoética & 1 \\
Estudos Descritivos sem Referencial Teórico Adotado & 13 \\
\hline Total & 46 \\
\hline
\end{tabular}

Quadro 1. Referencial teórico-metodológico das teses e dissertações avaliadas, Fortaleza, CE, 2008.

\begin{tabular}{|l|c|}
\hline Temáticas & $\%$ \\
\hline Paciente & 70 \\
Paciente e prontuário & 12 \\
Enfermeiro & 6 \\
Famíia & 4 \\
Documentos & 4 \\
Paciente e enfermeiro & 2 \\
Família e prontuário & 2 \\
\hline
\end{tabular}

Quadro 2. Temas das teses e dissertações avaliadas. Fortaleza, CE, 2008.

\begin{tabular}{|l|c|}
\hline Áreas & $\%$ \\
\hline Assistência de enfermagem no processo de diálise & 34 \\
Processo e teoria de enfermagem & 18 \\
Saúde da criança e do adolescente & 14 \\
Administração de fármacos & 9 \\
Saúde do idoso & 8 \\
Perfil epidemiológico dos pacientes & 8 \\
Cuidado familiar frente à Doença Renal Crônica & 4 \\
Assistência de enfermagem no transplante renal & 4 \\
Sexualidade do paciente renal & 2 \\
\hline
\end{tabular}

\section{Quadro 3. Áreas temáticas das teses e dissertações avaliadas. Fortaleza,} CE, 2008.

se trata de investigar um fenômeno tão novo como é o ensino ${ }^{(15)}$.

Desde então, muitas conQuistas aconteceram, os cursos de especialização foram expandidos por todo Brasil, isso devido à exigência do Ministério da Saúde com relação à capacitação técnica dos enfermeiros atuantes nos serviços de Terapia Renal Substitutiva. Além disso, a RDC no 154 de 2004 torna imperativo Que o enfermeiro Que esteja inserido nos serviços de diálise seja especializado em Enfermagem em Nefrologia, ou Que esteja em processo de capacitação $0^{(16)}$.

$\mathrm{O}$ desejo e as necessidades dos profissionais enfermeiros de fortalecer a categoria e conQuistar novos espaços deram origem à Associação Brasileira de Enfermagem em Nefrologia - SOBEN em $1983^{(17)}$. Esta associação é significativa no desenvolvimento do conhecimento em nefrologia, porQue favorece a inclusão dos enfermeiros Que trabalham na área, em grupos de estudos, pesquisas e especializações, isso também influenciou a ampliação conhecimento na área e a buscar por cursos de pós-graduação. Uma das coneuistas da SOBEN foi a participação na elaboração da portaria 2042 do Ministério da Saúde, Que determina o regulamento técnico para o funcionamento de serviços de TRS e suas normas a serem definidas em todo o país.

É evidente a supremacia da Região Sudeste (63\%) na produção desse conhecimento, en®uanto a Região Norte não apresentou nenhum estudo na área. A Região Sul apresentou 20\%, a Nordeste 13\% e a Centro Oeste 4\%. Resgatando informações do Ministério da Ciência e Tecnologia ${ }^{(18)}$ percebemos Que na última década há um incentivo maior de investimento em pesquisa no âmbito de pós-graduação, tanto em instituições federais como estaduais. Portanto, espera-se que haja uma ampliação dos cursos de pós-graduação stricto sensu em todas as regiões, em especial a região norte.

No Quadro 2 está demonstrado que os enfermeiros priorizaram trabalhar com o paciente em suas investigações. Em seguida, verifica-se Que os profissionais enfermeiros são escolhidos como os sujeitos dos estudos, demonstrando Que é necessário explorar suas experiências enQuanto atuantes nos serviços de diálise e transplante renal. A família também aparece com enfoque no processo investigativo.

Nesse sentido, o desafio atual dos profissionais é reconhecer e utilizar os relacionamentos Que as pessoas identificam como família ou externam como tal. É necessário repensar a família em termos de processo de interações entre pessoas e como elas constroem a noção de família num contexto múltiplo de raça, idade, gênero, preferência sexual, situação socioeconômica, etnicidade, localidade e historicidade. O trabalho com famílias reQuer habilidades do profissional para identificar a complexidade das relações Que se estabelecem nas famílias, por meio das interações entre seus próprios membros e deles com a comunidade ${ }^{(19)}$.

No Quadro 3 verifica-se que a assistência de enfermagem no processo dialítico foi a área temática mais pesquisada pelos enfermeiros em seus estudos. Esta área temática desvela a preocupação dos pesQuisadores com a população adulta, em investigações sobre Qualidade de vida, educação em saúde, acesso vascular para hemodiálise, adesão ao tratamento dialítico e dietético, relação enfermeiro e paciente, compreensão do fenômeno da cronicidade pelo enfermeiro, o cuidado recebido e 


\begin{tabular}{|l|c|}
\hline Modalidades & $\%$ \\
\hline Hemodiálise & 56 \\
Indefinido & 25 \\
Diálise peritoneal & 11 \\
Transplante renal & 5 \\
Conservador & 3 \\
\hline
\end{tabular}

Quadro 4. Modalidade terapêutica para IRCT. Fortaleza, CE, 2008.

\begin{tabular}{|l|c|}
\hline Aplicabilidade na enfermagem & $\mathbf{n}$ \\
\hline Consulta de Enfermagem & 1 \\
$\begin{array}{l}\text { Planejamento da assistência de enfermagem para um } \\
\text { cuidado de Qualidade }\end{array}$ & 7 \\
Educação em Saúde & 7 \\
$\begin{array}{l}\text { Cuidado integral } \\
\text { Planejamento da assistência de enfermagem para melhor } \\
\text { Qualidade de vida }\end{array}$ & 5 \\
$\begin{array}{l}\text { Compreensão do enfermeiro acerca da cronicidade } \\
\text { PesQuisa em enfermagem }\end{array}$ & 2 \\
$\begin{array}{l}\text { Aplicabilidade dos instrumentos de Qualidade de vida, SF- } \\
36 \text { e WHOQoL }\end{array}$ & 1 \\
$\begin{array}{l}\text { Reflexão da assistência de enfermagem para um cuidado de } \\
\text { Qualidade }\end{array}$ & 3 \\
$\begin{array}{l}\text { Gerenciamento do serviço de diálise } \\
\text { Planejamento da assistência de enfermagem pré-transplante } \\
\text { e pós-operatório }\end{array}$ & 1 \\
$\begin{array}{l}\text { Compreensão do enfermeiro acerca das modificações na } \\
\text { vida do idoso }\end{array}$ & 1 \\
\hline Total & 32 \\
\hline
\end{tabular}

Quadro 5. Aplicabilidade na enfermagem. Fortaleza, CE, 2008.

prestado na visão do enfermeiro e paciente.

O processo de enfermagem, en®uanto método para resolução de problemas e forma de prestar cuidados de enfermagem fundamentados com o uso de teorias de enfermagem ocorre nesta análise gráfica como a segunda área temática de maior interesse pelos profissionais. É relevante ressaltar Que essa forma sistematizada de cuidar teve representatividade na hemodiálise, diálise peritoneal e transplante renal. Aparece como teorias de enfermagem utilizadas, a teoria Humanística de Paterson e Zderad (1), a teoria do Autocuidado de Dorothea Orem (2), a teoria do Cuidado cultural de Madeleine Leininger (2), a teria do Alcance de Objetivos de Imogene King ( I), teoria da Adaptação de Callista Roy (I) e a teoria brasileira Sócio-Humanística (I) de Capella e Leopardi.

Expor esta informação é de grande relevância, pois desde Wanda Aguiar Horta, em 1970, a tentativa de implantação dessa atividade tem sido relevante, porém ainda não substancial na Enfermagem Brasileira. A sua árdua construção e implementação, a dificuldade de manutenção, Quando não obstante, a sua ausência em muitos serviços de Enfermagem, vêm sendo destacadas por vários estudos ${ }^{(20-24)}$.

Dentro deste contexto, percebe-se a atuação profissional de enfermagem em nefrologia no setor de diálise renal, ainda tímida na realidade da sistematização da assistência de enfermagem (SAE). Em estudo realizado ${ }^{(23)}$ constatou-se uma escassa produção científica publicada em periódicos brasileiros na área, talvez refletindo as diminutas experiências da aplicação dessa metodologia nos serviços hospitalares e satélites Que prestam este tipo de terapêutica ao paciente renal crônico.

Em análise do Quadro 4, constata-se Que a hemodiálise foi a modalidade terapêutica para o paciente com IRCT mais investigada pelos pesouisadores, seguida da diálise peritoneal, contudo destaca-se o fato da modalidade terapêutica indefinida com 18\% Que vela a possibilidade de sabermos oual a modalidade investigada pelos estudos.

O tratamento conservador aparece como contexto no cuidado à saúde da criança e do adolescente, visando o planejamento da assistência de enfermagem para uma melhor Qualidade de vida a essa população. $\mathrm{O}$ transplante renal (Tx-renal) surge como cenário para a realização da consulta de enfermagem e para educação em saúde.

Ao observar o Quadro 5, tem-se uma visão da contribuição dessas pesQuisas para a enfermagem, Quando nele se elencam aplicabilidades de diversas ordens.

Percebe-se que o cuidado está presente implícita ou explicitamente em todas as finalidades do estudo. Um cuidado integral, previsto pela Portaria № 1.168 de 15 de junho de 2004 Que institui a Política Nacional de Atenção ao Portador de Doença Renal ${ }^{(25)}$, um cuidado familiar, um cuidado domiciliar Que permeiam as atividades de assistência nefrológica a esses pacientes.

É perceptível Que todas essas aplicabilidades visem uma melhoria da assistência de enfermagem, com uma amplitude de ações Que envolvem a educação em saúde, a aplicação de instrumentos de eualidade de vida com o SF-36 e o WHOQoL, este último específico para o paciente renal crônico, a consulta de enfermagem sistematizada e embasada teoricamente e por último, a pesQuisa em enfermagem a favorecer terreno fértil para a produção científica elevando a profissão enQuanto ciência, na perene construção e reconstrução de saberes e prática.

A categoria alteração do estado de saúde tem atributos essenciais que firmam a natureza da doença renal crônica. Descreve conceitos relacionados à irreversibilidade da condição de doente, ao seu aparecimento lento, progressivo e incurável, e a outras morbidades pré-existentes além da hereditariedade ${ }^{(26)}$. Dentro deste contexto é importante ressaltar Que há preocupação do enfermeiro em compreender o fenômeno da cronicidade.

A organização do processo de trabalho em enfermagem revelase como uma preocupação desses estudos. Os enfermeiros almejam uma reflexão da assistência de enfermagem, bem como o seu planejamento para a presteza de um cuidado de Qualidade, seja no âmbito da saúde da criança ou do adolescente, adulto ou idoso, 
ou no cenário da diálise e transplante renal.

Dessa forma, percebe-se que a amplitude de possibilidades de produção de conhecimento na enfermagem em nefrologia, revelando interesse em diversas áreas temáticas, ao refletir nos estudos analisados o desenvolvimento de atividades das mais variadas preocupações.

\section{CONCLUSÃO}

Infere-se através desta análise, Que a temática insuficiência renal crônica ainda é pouco abordada nos cursos de pós-graduação stricto senso, entretanto, há índicos de expansão dos estudos científicos nesta área desde a década de noventa. Além disso, o estudo possibilitou identificar os marcos científicos Que norteiam o desenvolvimento das pesquisas na área de enfermagem em nefrologia, possibilitando identificar a tendência de "pluralidade" de métodos como um caminho de construção do conhecimento em saúde e Enfermagem. As dissertações e teses estão sendo desenvolvidas nos diversos níveis de atenção à saúde. Dessa forma, as Questões relacionadas ao cuidado de enfermagem na promoção e prevenção, assim como, na cronicidade e reabilitação no transplante renal, foram contempladas nos diferentes ciclos desenvolvimentais.

Percebeu-se ainda, Que a enfermagem vem ampliando a compreensão do seu foco de atenção, contemplando no cuidado clínico a subjetividade do ser humano no seu processo saúdedoença, levando em consideração as Questões sociais, históricas e fenomenológicas.

\section{REFERÊNCIAS}

1. Barreira IA, Batista SS. O movimento de reconsideração do ensino e da pesquisa em história da enfermagem. Rev Bras Enferm 2003; 56(6): 702-6.

2. Silveira LC, Dias MAS, Chagas MIO, Damasceno MMC, Fraga MNO. Tendências das teses de doutoramento em enfermagem produzidas na Universidade Federal do Ceará. Texto Contexto Enferm 2003; 12(3): 314-22.

3. Padilha MICS, Kletemberg DF, Gregório VRP, Borges LM, Borenstein MS. A produção da pesquisa histórica vinculada aos programas de pós-graduação no Brasil, 1972 a 2004. Texto Contexto Enferm 2007; 16(4): 67 I-9.

4. Reibnitz KS, Prado ML. Inovação e Educação em Enfermagem. Florianópolis: Cidade Futura; 2006.

5. Sociedade Brasileira de Nefrologia. Notícia. São Paulo; 2003. [citado em: 20 out 2008]. Disponível em: http:// www.sbn.org.br/notícias

6. Sociedade Brasileira de Nefrologia. Censo. São Paulo; 2007. [citado em: 20 out 2008]. Disponível em: http:// www.sbn.org.br/censo/2008

7. Almeida AM, Oliveira ERA, Garcia TR. Pesquisa em Enfermagem e o positivismo. Rev Esc Enferm USP 1996; 30 (1): 25-32.

8. Barros E, Manfro R, Thomé E, Gonçalves LF. Nefrologia: rotinas, diagnóstico e tratamento. Porto Alegre: Artmed; 2006.

9. Gil AC. Como elaborar projetos de pesQuisa. São Paulo: Atlas; 2007.

10. Associação Brasileira de Enfermagem. Centro de Estudos e Pesquisa em Enfermagem. Catálogos disponíveis em CD-ROM: CD 75 Anos da ABEn - Vol I - 8 (1979 - 2000); CD 80 Anos da ABEn - v 19-24 (2001-2005). Catálogos de 2006 e 2007 [citado em: 15 nov 2008]. Disponível em: http:// www.abennacional.org.br

11. Demo P. Metodologia do conhecimento científico. São Paulo: Atlas; 2008.

12. Turato ER. Tratado da metodologia da pesquisa clínicoQualitativa. Petrópolis: Vozes; 2003.

13. Leopardi MT. Teoria e método em assistência de enfermagem. Florianópolis: Soldasoft; 2006.

14. Ministério da Ciência e Tecnologia (BR). Indicadores sobre o ensino de pós-graduação. Brasília; 2006. [citado em: 23 out 2008]. Disponível em: http://www.mct.gov.br
15. Guedes GF, Ohara CVS, Silva GTR, Franco GRRM. Ensino clínico na enfermagem: a trajetória da produção científica. Rev Bras Enferm 2009; 62(2): 283-6.

16. Ministério da Saúde (BR). Agência Nacional de Vigilância Sanitária - ANVISA. RDC no. 154, de 15 de junho de 2004 (versão republicada em 2006). Brasília; 2006. [citado em: 15 nov 2008]. Disponível em: http://e-legis.anvisa.gov.br/leisref/ public/showAct.php?id=22875\&word

17. Fortes RMSO. $\mathrm{O}$ fortalecimento da enfermagem em nefrologia e o trabalho da Associação Brasileira de Enfermagem em Nefrologia - SOBEN. Nursing 1999; I5(2): 10-11.

18. Ministério da Ciência e Tecnologia (BR). Recursos aplicados na pós-graduação. Brasília; 2006. [citado em: 23 out 2008]. Disponível em: http://www.mct.gov.br/index.php/content/view/ 27959.html

19. Filizola CLA, Ribeiro MC, Pavarini SCI. A história da família de Rubi e seu filho Leão: trabalhando com famílias de usuários com transtorno mental grave através do Modelo Calgary de Avaliação e de Intervenção $\mathrm{n}$ a família. Texto Contexto Enferm 2003; 12(2).

20. Carvalho EC, Bachion MM, Dalri MCB, Jesus CAC. Obstáculos para a implementação do processo de enfermagem no Brasil. Rev Enf UFPE [periódico online] 2007 Jul [citado em: 20 nov 2008]; 10(1):[4 páginas]. Disponível em: http://www.ufpe.br/ revistaenfermagem/index.php/enfermagem/article/view/42/26

21. Kletemberg DF, Siqueira MD, Mantovani MF, Jesus CAC. Uma história do processo de enfermagem nas publicações da Revista Brasileira de no período 1960-1986. Esc Anna Nery Rev Enferm 2006; 10(3): 478-86.

22. Takahashi AA, Barros ALBL, Michel JLM, Souza MF. Dificuldades e facilidades apontadas por enfermeiras de um hospital de ensino na execução do processo de enfermagem. Acta Paul Enferm 2008; 2 I ( I): 32-8.

23. Figueiredo RM, Zem-Mascarenhas SH, Napoleão AA, Camargo AB. Caracterização da produção do conhecimento sobre sistematização da assistência de enfermagem no Brasil. Rev Esc Enferm USP 2006; 40(2): 299-303.

24. Backes DS, Esperança MP, Amaro AM, Campos IEF, Cunha ADO. Sistematização da assistência de enfermagem: percepção dos enfermeiros de um hospital filantrópico. Acta Sci Health Sci 2005; 27(1). 
Pennafort VPS, et al.

25. Ministério da Saúde (BR). Portaria no ${ }^{\circ}$ I.168/GM, de 15 de junho de 2004, institui a Política Nacional de Atenção ao Portador de Doença Renal. Brasília; 2004. [citado em: 23 nov 2008]. Disponível em: http://bvsms.saude.gov.br/bvs/
publicacoes/portaria_I I68_ac.htm

26. Kusumota L, Rodrigues, RAP, Marques S. Idosos com insuficiência renal crônica: alterações do estado de saúde. Rev Latino-am Enfermagem 2004; 12 (3): 525-32. 